\title{
Improving core medical training - innovative and feasible ideas to better training
}

\author{
Authors: Fiona Tasker, ${ }^{A}$ Peter Dacombe, ${ }^{B}$ Andrew F Goddard ${ }^{C}$ and Bill Burr ${ }^{D}$
}

\begin{abstract}
A recent survey of UK core medical training (CMT) training conducted jointly by the Royal College of Physicians (RCP) and Joint Royal College of Physicians Training Board (JRCPTB) identified that trainees perceived major problems with their training. Service work dominated and compromised training opportunities, and of great concern, almost half the respondents felt that they had not been adequately prepared to take on the role of medical registrar. Importantly, the survey not only gathered CMT trainees' views of their current training, it also asked them for their 'innovative and feasible ways to improve CMT'. This article draws together some of these excellent ideas on how the quality of training and the experience of trainees could be improved. It presents a vision for how CMT trainees, consultant supervisors, training programme directors, clinical directors and managers can work together to implement relevant, feasible and affordable ways to improve training for doctors and deliver the best possible care for patients.
\end{abstract}

KEYWORDS: Improving training, core medical training, innovative ideas

\section{Introduction}

'The long term delivery of high quality care depends on doctors receiving excellent training.' ${ }^{1}$

Recent work by several organisations, and feedback from medical trainees, has raised concerns over the effectiveness and quality of current training. ${ }^{2,3}$ To explore this issue, the Royal College of Physicians (RCP) and Joint Royal College of Physicians Training Board (JRCPTB) undertook a survey of core medical training (CMT) trainees in the UK in June 2013. The survey gathered trainees' views of key aspects of CMT. It identified three areas that need urgent attention. ${ }^{4}$

Authors: Acore medical trainee year 2 and clinical fellow to the president 2013-2014, Royal College of Physicians, London, UK; Btrauma and orthopaedic surgery ST4 and clinical fellow at the GMC 2013-2014, London, UK; Cregistrar, Royal College of Physicians, London, UK; D professor and previous medical director, Joint Royal College of Physicians Training Board, London, UK (i) Time for training: the demands of providing service have led to considerable loss of training opportunities, with trainees reporting that attendance at outpatients and formal teaching sessions was seriously compromised. Trainees spend a lot of this service time doing menial tasks and over $90 \%$ report that service takes up $80-100 \%$ of their time.

(ii) Appropriate supervision: clinical and educational supervision is variable, with trainees sometimes getting little consultant feedback on their clinical performance.

(iii) Perception of, and preparation for, a career in medicine: $44 \%$ of CMT 2 trainees reported that CMT has not prepared them to be a medical registrar.

The Charter for Postgraduate Medical Training (developed by the Royal College of Physicians of Edinburgh), outlines several priorities, including the appropriate balance between service provision and learning, adequate induction, supervision and continuing support, and leadership and management experience. ${ }^{5}$ To explore these issues further and to improve our knowledge of trainee perceptions, the RCP/JRCPTB survey not only gathered CMT trainees' views of their current training, but also gave the opportunity for trainees to suggest ways of improving CMT. Of the $871 \mathrm{CMT}$ trainees in the UK who responded to the survey (a 32\% response rate), 269 chose to comment in a free-text box that sought the best 'innovative and feasible ways to improve CMT'. Here, we present the challenges highlighted by the RCP/JRCPTB survey, and suggest ways in which consultant supervisors, training programme directors, clinical directors, managers and CMT trainees themselves can work together to improve CMT. The examples given in Boxes 1-4 are intended to demonstrate how adjustments to CMT programmes could improve the quality of CMT training with minimal resource implications.

\section{Solutions that individuals responsible for delivering CMT can implement}

Challenge 1 - time for training and the pressures of service provision

Service must 'be focused around patient needs, but the work undertaken by doctors in training should support learning wherever possible.' CMT trainees provide a crucial service to medical inpatients and the acute medical take. However, in a recent RCP survey of members and fellows, $75 \%$ stated that their main professional concern was clinical staff shortages. ${ }^{6}$ It 
is often trainees who feel the brunt of these service pressures, ${ }^{7}$ which can limit opportunities to focus on personal educational development. For example, trainees reported being unable to attend training opportunities, such as local and regional teaching sessions, training in procedural skills and outpatient clinics. ${ }^{4}$ In other instances, CMT trainees reported performing routine ward tasks, which are often non-medical or similar to those at foundation level, which contribute both to work pressure and to the feeling of lack of progression during CMT. It is important not to underestimate the valuable contribution that service provision can make to training, provided that it is supplemented by time to train and by opportunities for structured feedback and reflection. It is important to ensure that trainees receive the right balance between delivering care for patients (with all the opportunities for development that that presents) and having the opportunity to develop their skills and practice in more formal educational environments. 'Training and ways of working have to change to cope with a more flexible workforce. If not, we risk losing many talented, dedicated and skilled doctors' ${ }^{8}$

\section{Solution 1 - rota design}

Rotas must be designed with training needs in mind and not just reflect service requirements. They must include protected time for training. Junior doctors have an important role in helping to facilitate rota changes, but need to be supported by consultants and human resources to design rotas that take account of core training opportunities, with cover arrangements clearly identified so that trainees do not miss these valuable sessions. Core training opportunities include: designated outpatient clinics; bleep-free regional and local teaching sessions; and simulation training, including skills lab. On-call shift and working patterns should be adjusted to enable the following.

> Trainee attendance at the post-take ward round so that they can present patients they have clerked and receive feedback from consultants. This would also assist with the continuity of care of patients, and inevitably involves changes to the timing of post take rounds.

$>$ Time to shadow the on-call registrar/step up as a registrar with support (Box 1).

\section{Box 1. Acting up as a medical registrar with support.}

The survey has shown that trainees want to have increased responsibility towards the end of CMT and look to 'act up' as much as possible. 'Acting up' should enable CMT trainees to experience the transition of becoming a medical registrar in a supported environment with a designated supervisor who is always available for support, including out of hours or during on-call work. This would be most appropriate during the last 4 months of CMT or when trainees have gained MRCP. For those trainees who wish to gain this experience, there should be phased developments of holding the medical registrar bleep during the day when other senior colleagues are around, leading on to night-time responsibilities but always with appropriate back-up. Trainees could have day-time responsibility, including observed ward rounds. A local policy should be in place and followed.

$\mathrm{CMT}=$ core medical training .

\section{Box 2. Working with the same team.}

Same-team working should be encouraged as much as possible, and was recommended by the Future Hospital Commission. ${ }^{9}$ Working in blocks of on-calls of 6 months instead of 4 months could facilitate apprenticeship-style training and, depending on the type of trust, could prevent on-calls from disrupting ward work. These changes would be likely to encourage consultants and registrars to teach trainees in their team as they get to know them better. The continuity of working with the same team members would improve morale, particularly for the trainees. It would be rewarding for supervisors to observe trainees as they develop skills over time and supervisors will be better able to identify and support trainees in difficulty.

$>$ Time for personal study to allow for reflection and e-Portfolio entries, study for exams or pursuing research and/or quality improvement.

> Blocks of acute medicine with the same team on-call to encourage continuity of care and improved teamwork (Box 2).

Solution 2 - differentiation between FY2 and CMT posts One way to tackle the feeling of lack of progression from foundation years is making sure the CMT trainee is not the most junior member of the medical team. If they are, the workforce should be configured so that non-medical tasks, such as phlebotomy, are dealt with appropriately by non-medical staff. CMT trainees should have extended roles in the 'day job' setting, such as leading ward rounds and gaining experience of decision making under direct consultant supervision, supervising junior trainees, attending outpatient clinics and working closely with the specialty registrar to manage some referrals. Extended roles on-call could include leading the resuscitation team and taking referrals in a supported setting.

\section{Challenge 2-CMT trainers}

The Royal College of Physicians of Edinburgh Charter for Medical Training states that 'Trainers are selected and appropriately trained for their role and have a suitable job plan, appropriate workload and time to develop trainees'. ${ }^{4}$ Trainees reported that educational and clinical supervisors appeared not to have enough time to train and, further, that many trainers did not have adequate training on the use of the e-Portfolio. Many trainees experienced frequent changes of educational supervisors throughout the programme, which is not ideal for continuity of training. ${ }^{4}$

\section{Solution-educational supervisors}

We suggest that educational supervisors:

$>$ must be trained appropriately and receive support for the role

$>$ should have their training responsibilities recognised in their job plan and the time that trusts or local health boards have allocated must be devoted to training activity

> should be allocated to a trainee for a minimum of 12 months with the option for 2 years if possible (and agreed with the trainee)

$>$ ideally, trainee preference should be taken into account in the allocation of supervisors 
$>$ the development of local and regional awards for best supervisors and educators would celebrate excellence and could motivate supervisors to perform better.

In addition, JRCPTB supports the idea that trainees should give feedback on the quality of educational supervision, and endorses the use of a survey tool designed for the use of local training programme directors. In addition, JRCPTB is supportive of a feedback system under development by doctors in Health Education Northern whereby Annual Review of Competence Progression (ARCP) panels are asked to assess the quality of educational supervisors' reports, and for this to be fed back to supervisors.

\section{Challenge 3 - developing better support for CMT trainees}

Many CMT trainees report low morale, feel undervalued in their roles and feel that they lack support in their jobs. ${ }^{4}$ Most trusts and local health boards did not manage to preserve traditional 'firm' structures when the European Working Time Regulations led to widespread shift working. Trainees lost the peer and senior support and continuity that were part of the firm system. There must be local support for trainees in their day-to-day work, as well as to assist with educational training opportunities, career guidance and to highlight issues affecting their training.

\section{Solution 1 - departmental, trust and regional induction} CMT induction is vital if trainees are to understand exactly what is expected of them, as well as their rights as a core medical trainee, what the programme should provide and how best to make the most of their two-year training scheme. CMT programmes must have a mandatory induction process, with clarity of the expectations required from trainees, what must be achieved to progress, ARCP requirements, and how to use the e-Portfolio system as well as who's who at local education and training boards (LETB) and/or deanery, local health board level and trust, including the key people to go to for information or advice. It might be beneficial for previous CMT trainees, who have progressed to become medical registrars, to meet the incoming trainees, to reflect on their own experience of CMT, the key lessons they learned and how they would approach it differently if they had their opportunity again.

\section{Solution 2 - local CMT trainees' committee and CMT trainee representative}

Each hospital should have a CMT representative who should feedback problems to the LETB and/or deanery either directly or via a regional CMT representative. It should be the responsibility of the CMT representative in each hospital to set up a regular and active trainees' committee, and they should receive consultant support to help them achieve this. The college tutor, associate college tutor, or other designated consultant should have responsibility for the educational and pastoral support of CMT trainees. CMT trainees should also have a representative on the trust educational committee. As Robert Francis commented, trainees are often aware of problems that might be hidden from others. ${ }^{10}$

\section{Solution 3-mentoring scheme}

Mentoring is the practice of facilitating development.

Mentors provide support, direction and an objective view on how the mentee can develop and progress in their working environment. ${ }^{11}$ It was frequently suggested that CMT trainees would benefit from having an assigned mentor, either a registrar, or consultant who was in tune with both CMT training, and the medical training pathway beyond this. Many trainees felt that this would offer them much needed guidance regarding their training, as well as support and career advice for the future. If a mentoring scheme is not already in place for doctors, then setting up a local mentoring programme could be considered.

\section{Solution 4- 'CMT trainee advocate'}

Appointment in each hospital of a 'CMT trainee advocate' would be beneficial. An advocate would be cognisant of the curriculum, understand the expectations of the RCP and be available and accessible for trainees to raise concerns in confidence. For example, they could run a drop-in clinic for trainees. The CMT trainee advocate could ensure that trainees' concerns are collated and passed onto the trust or health board directorate and to the LETB and/or deanery.

\section{Solution 5 - careers guidance}

There should be a careers lead in each hospital, LETB and/ or deanery who organises information on careers and gives guidance as how to prepare for ST3 applications. Whenever possible, trainees should be offered the opportunity to choose an educational supervisor in a preferred specialty for career advice. Where resources allow, a taster week could be an option for trainees to explore career aspirations.

\section{Solution 6 -create and protect training opportunities}

One example of gaining experience of decision making while under direct consultant supervision on the wards includes a trainee taking the lead on a ward round followed by feedback and teaching on the cases reviewed. In addition to apprenticeship-style learning, protected time should be allocated for local and regional teaching sessions that are bleepfree. Trainees in one LETB commented on the advantage related to the fact that teaching sessions in all trusts were 'in phase' so that a curriculum could be followed through different trusts. CMT trainees want interactive sessions that are practical and focused on passing MRCP(UK) Part 2 Clinical Examination (PACES) and fulfilling the CMT curriculum. Recommended teaching topics include the scenarios that CMT trainees are faced with when on-call, updates on latest guidelines, medical emergencies and/or procedural skills, and managing patients in the outpatient setting. A teaching session on a case delivered by a consultant before working hours could be organised so that all staff in the hospital have the opportunity to discuss interesting educational cases. Trainees must be supported to attend training opportunities, including being able to take study leave for relevant courses.

\section{Challenge 4 - valuing trainees}

The RCP/JRCPTB survey indicated that morale is low. ${ }^{4}$ This seems to be partly because trainees feel overworked and undervalued and because they are unable to plan their lives in advance because of late release of rotas and a lack of choice in their rotations. 


\section{Recommendations}

$>$ An outline of the rotations on offer should be available at the time that CMT posts are advertised. Ideally, trainees should be able to choose between the rotations on offer, and trainee choice could be taken into account in deciding the CMT2 rotations. In reality, recruitment will depend on ranking to allocate rotations so trainees should be able (when possible), to swap rotations while maintaining the acute take/nonacute balance via a trust swap shop for example, to improve ownership of their training.

> On-call rotas should be made available in good time. We are aware of instances where trainees have been given as little as 2 weeks' warning and sometimes even less than this.

> Other things that can make trainees feel valued include:

- a consultant taking their junior team on a coffee break after the ward round to debrief and help with prioritising jobs

- having an annual regional event whereby trainees are given recognition and rewarded for excellent work in audit and/or quality improvement projects for example, creating incentives to achieve more than the standard requirements.

\section{Solutions that CMT trainees can implement}

'Experience shows that with the right kind of guidance, trainee doctors are well placed and suited to catalysing and sharing innovation across the NHS in a way that engages other professional groups in change agency'. ${ }^{12}$ Trainees must be empowered to take ownership of CMT and their own training. CMT trainees should be supported to undertake projects designed to improve CMT training, which will also shape the future of CMT training. As demonstrated by this survey, trainees have a valuable contribution to make when designing and delivering improvements to their own training.

The following challenges are linked with suggestions of how core medical trainees can improve their training, made by trainees themselves.

\section{Challenge 5 - experience of outpatient clinics}

Experience with reviewing patients in an outpatient setting in CMT is important preparation for becoming a registrar, when they will be expected to hold clinics more independently. Of the trainees surveyed, $89 \%$ found attending outpatient clinics either valuable or very valuable, but $81 \%$ of trainees reported that they were frequently prevented from attending outpatient clinics because of ward commitments. ${ }^{4}$

\section{Recommendations}

A list of all clinics could be made available on a shared drive so that trainees could sign up to a variety of clinics in advance in addition to factoring in time for attending outpatient clinics in the rota (Box 3).

\section{Challenge 6 - exposure to procedural skills and simulation}

Trainees have expressed that it can be difficult to gain exposure to some clinical procedures, such as central venous cannulation

\section{Box 3. Attending outpatient clinics.}

CMT trainees should not feel pressured to stay on the wards and miss learning opportunities in clinic. Time to attend outpatient clinics should be mandatory and built into the rota from the start of the rotation, which should allow enough time to arrange for appropriate ward cover. This could be one clinic once a week or a 1-week block every 2 months when trainees would have no other clinical commitments. Supervising consultants and registrars should support and encourage CMT trainees to attend clinic. There should ideally be a consultation room for the trainee and time factored in for consultant supervision so that trainees receive feedback and the opportunity to learn and get work place-based assessments. The trainee's clinic letters should also ideally be reviewed by the consultant leading the clinic and feedback given. Trainees could create a log of patients reviewed anonymously as evidence of learning.

$\mathrm{CMT}=$ core medical training.

and inserting intercostal drains, because the amount of experience gained is often dependent on which specialities the trainee has been allocated to, for example trainees in the intensive treatment unit (ITU) are likely to gain more experience in these procedures. ${ }^{4}$

\section{Recommendations}

$>$ Swap shop of skills training (Box 4).

$>$ Encourage consultants who have a dedicated list of procedures to invite CMT trainees along via a sign-up sheet.

> CMT trainees could have a rota to hold a baton bleep for procedural training. Whenever a procedure with training value is to be carried out in the hospital, the trainee is bleeped and is expected to attend to either perform the procedure under supervision or observe.

> Liaise with ITU physicians, anaesthetists and radiologists to organise procedural skills teaching, including using ultrasound.

$>$ Organise a taster and/or study leave of several weeks on ITU to gain experience with procedural skills and of handling sick patients and procedures.

\section{Box 4. Swap shop of skills training set up locally.}

To facilitate peer-to-peer teaching of practical skills, a swap shop of skills training could be set up locally by CMT and specialist registrar trainees in different specialties. For example, on neurology, a trainee might perform 5-10 lumbar punctures per week, of which some are often predictable. By the end of the rotation, that trainee would be competent in this skill, but might have never inserted a central line or put in a chest drain. If there was a local website on the intranet where trainees could log these procedures sessions and bleep numbers, competent trainees could teach their peers to perform procedures ideally with registrar supervision. This would result in CMT trainees gaining fairer access to skills training.

CMT = core medical training. 


\section{Box 5. How are the JRCPTB and RCP working to improve the quality of CMT?}

\section{JRCPTB}

> The Committee for CMT is a subcommittee of the JRCPTB comprising training programme directors from all training schemes in the UK, and with trainee and lay representation. The role of the committee is to produce the curriculum and assessment system for CMT for approval by the GMC. They also advise on the quality and delivery of training. They have achieved improvements in the governance and consistency of training and assessment, and are contributing to developing a set of quality criteria.

> JRCPTB, working with HEE, have initiated an expert working group to include simulation training in the CMT curriculum.

$>$ JRCPTB have been working to improve work place-based assessments for trainees and trainers, and have a programme of development for e-Portfolio, including the introduction of more online forms and e-learning alignment with curriculum to guide and facilitate access for trainees.

> JRCPTB is developing a set of quality criteria to assure CMT programme quality, and is working with the GMC, HEE and the CMT committee to achieve this. The GMC appreciates the importance of this work and has agreed that additional CMT questions relevant to quality standards can be included in the national trainee survey.

\section{RCP}

> LTMD is about empowering junior doctors to learn and develop skills in quality improvement and putting these new skills into practice to make a real difference to their patient care. ${ }^{13}$ Quality improvement projects represent a realistic and dynamic process that enable trainees to bring about a visible change to their training environment. Resources are available to assist with setting up a quality improvement project. ${ }^{13,14}$ The LTMD programme supports trainees to implement change and LTMD hosts an annual quality improvement annual conference, which recognises excellent work and offers the opportunity to exchange ideas.

> The RCP are piloting a national mentoring scheme for CMT trainees, which launched in October 2014. This offers an exciting opportunity for junior doctors to learn from the experience of specialty trainees and consultants who have learnt the lessons and made the decisions that they will face in the coming years.

$>$ The RCP education department provides education and training initiatives. They organise, design and deliver programmes, including conferences, non-clinical workshops and Masters-level courses. They produce and provide access to multimedia resources, including e-resources, through their dedicated Medical Education Resource Centre. ${ }^{15}$

> The RCP careers department organises events to support CMT trainees with career choice and development such as the Medical Careers day.

> RCP members gain access to: Clinical Medicine and the CME section and 'Teach Ins' online and the opportunity to attend these sessions in person, which assist with self directed learning; and Best Practise to assist with practising evidence-based medicine.

$\mathrm{CMT}=$ core medical training; GMC = General Medical Council; HEE = Health Education England; JRCPTB = Joint Royal Colleges of Physicians Training Board; LTMD = Learning To Make a Difference.

$>$ Setting up a formal procedural skills training course, including simulated patients.

$>$ Consider a dedicated procedures area within the hospital and rotate CMTs through this area regularly.

\section{Challenge 7 - preparation to be a medical registrar}

Of the CMT2 trainees surveyed, $44 \%$ reported that CMT has not prepared them to be a medical registrar. Trainees clearly need better preparation for becoming a medical registrar.

\section{Recommendations}

> Opportunity to lead ward rounds once a week and gain feedback from the consultant.

$>$ Arrange to shadow the medical registrar.

$>$ Arrange to act up as a medical registrar with support (Box 1).

\section{Conclusion}

The JRCPTB and RCP play a role in influencing medical education and training and are concerned to improve the experience of CMT (Box 5). CMT trainees across the country have shared multiple innovative and feasible ways that could not only improve CMT training, but might also be applicable to aspects of training for all hospital doctors. The suggested solutions to improve CMT can be, and in some cases have already been, implemented by CMT trainees working with individuals responsible for delivering CMT. CMT trainees should be empowered to identify the problems with training in their hospitals and work with senior colleagues to put these ideas into practice. There are resources available to assist with setting up a quality improvement project. ${ }^{13,14}$ We are keen to support trainees and supervisors to improve CMT and, therefore, encourage anyone implementing a quality improvement project involving ideas that have been recommended in this article to register their project with Learning To Make a Difference at LTMD@rcplondon.ac.uk to receive a certificate to demonstrate their involvement in quality improvement as evidence of leadership.

\section{References}

1 Academy of Medical Royal Colleges. Value of the doctor in training. A charter for postgraduate medical training. London: AMRC, 2014.

2 Collins J. Foundation for excellence: an evaluation of the foundation programme. London: Medical Education England, 2010. 
3 Chauduri E, Mason N, Newbery N, Goddard A. Career choices of junior doctors: is the physician an endangered species? Clin Med 2013;13:330-5.

4 Tasker F, Newbery N, Burr B, Goddard A. Survey of core medical trainees in the United Kingdom 2013 - inconsistencies in training experience and competing with service demands. Clin Med 2014;14:149-56.

5 Royal College of Physicians of Edinburgh. Charter for medical training. Edinburgh: RCPE, 2011.

6 Royal College of Physicians (2014). Results from RCP research panel (Unpublished data).

7 Royal College of Physicians. The medical registrar: empowering the unsung heroes of patient care. London: RCP, 2013.

8 Royal College of Physicians. Hospital workforce. Fit for the future? London: RCP, 2013.

9 Future Hospital Commission. Future hospital: caring for medical patients. A report from the Future Hospital Commission to the Royal College of Physicians. London: RCP, 2013.

10 Francis R. The Mid Staffordshire NHS Foundation Trust Inquiry. Robert Francis inquiry report into Mid-Staffordshire NHS Foundation Trust. London: Department of Health, 2010.
11 British Medical Association, 2014. Available online at http://bma. org.uk/developing-your-career/career-progression/mentoring-andcoaching [Accessed 3 October 2014].

12 Penlington C, Marshall P. Why junior doctors are innovation leaders. Health Service J 2013:12 July.

13 The Royal College of Physicians, 2014. Available online at www. rcplondon.ac.uk/projects/learning-make-difference-ltmd [Accessed 2 October 2013].

14 Tasker F. How to lead a quality improvement project. Available online at http://careers.bmj.com/careers/advice/view-article. html?id=20010482 [Accessed 20 October 2014].

15 Royal College of Physicians, 2014. Available online at www. rcplondon.ac.uk/cpd [Accessed 2 October 2014].

Address for correspondence: Dr F Tasker, Royal College of Physicians, 11 St Andrews Place, Regents Park, London NW1 4LE, UK.

Email: fionatasker@doctors.org.uk

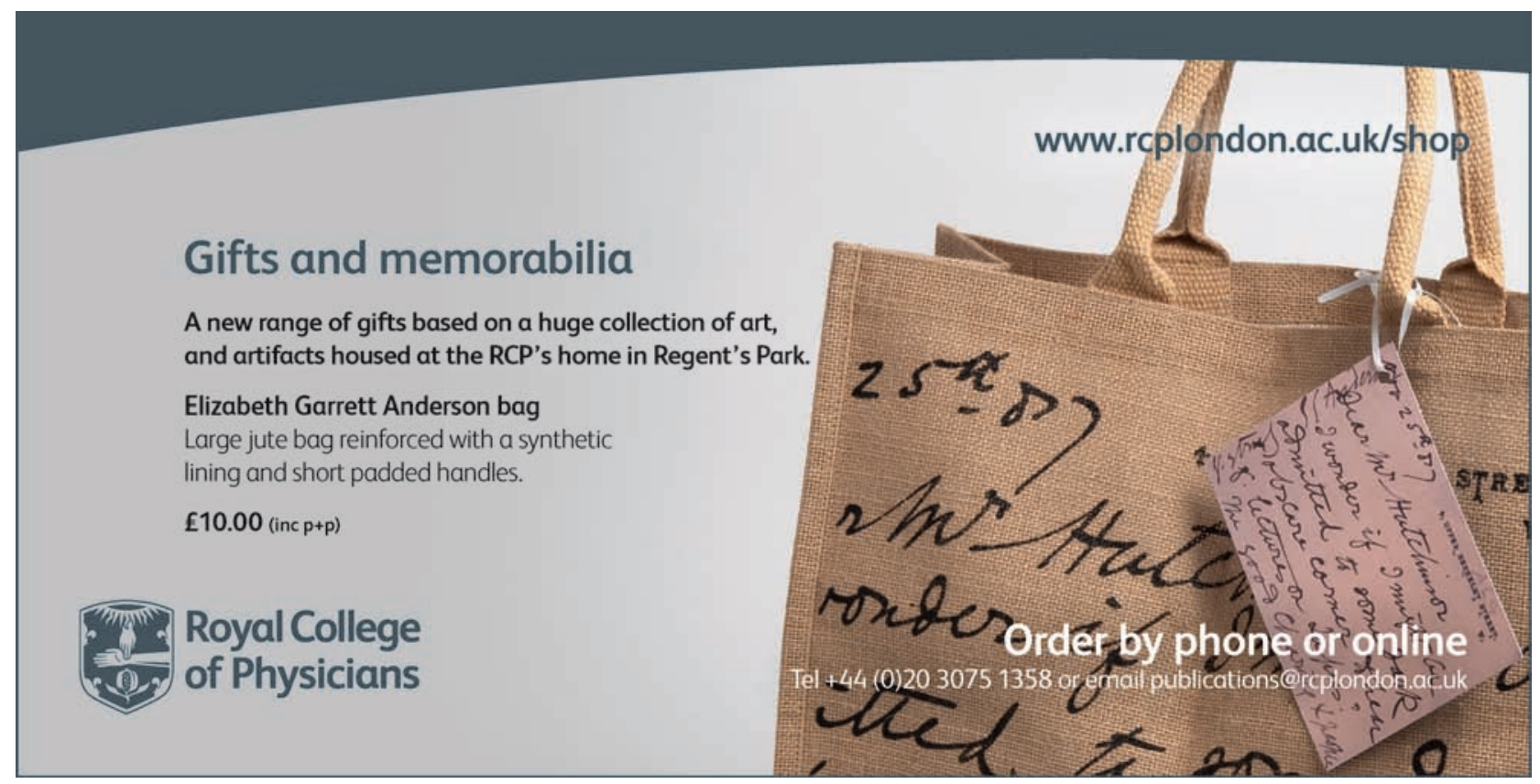

